\title{
招請講演 4
}

\section{TISSUE ENGINEERING, STEM CELLS AND CLONING:} APPLICATIONS IN UROLOGY

\author{
Department of Urology, Children's Hospital and Harvard Medical School, USA \\ Anthony Atala
}

Congenital abnormalities, cancer, trauma, infection, inflammation, iatrogenic injuries and other conditions may lead to genitourinary organ damage or loss, requiring eventual reconstruction. The use of native non-urologic tissues in the genitourinary tract is common, due to a lack of a better alternative, despite the known possible adverse effects.

Selective cell transplantation is providing a means to engineer genitourinary tissues that may be used for reconstruction. Tissue engineering follows the principles of cell transplantation, materials science and engineering towards the development of biological substitutes which would restore and maintain normal function.

Tissue engineering may involve matrices alone, wherein the body's natural ability to regenerate is used to orient or direct new tissue growth, or the use of matrices with cells. Both synthetic and natural biodegradable materials have been used, either alone, or as cell delivery vehicles.

Cells for tissue reconstitution can be derived from the native organ to be replaced, wherein a small biopsy of tissue is obtained, the specimen is dissociated, and the cells grown and expanded in culture. However, in situations where normal native tissue is not available, different stem cell sources may be explored. In addition, recent advances in the field of cloning have made it possible to retrieve cells using nuclear transfer techniques.

Tissue engineering has been applied experimentally in the field of urology for the reconstitution of several tissues and organs, including bladder, ureter, urethra, kidney, testis and genitalia. Fetal applications have also been explored. Recently, several tissue engineering technologies have been used clinically, including the use of cells as bulking agents for the treatment of vesicoureteral reflux and incontinence, urethral replacement, and bladder reconstruction. Recent progress suggests that engineered urologic tissues may have clinical applicability in the future. 